\title{
Mind wandering while reading easy and difficult texts
}

\author{
Shi Feng • Sidney D'Mello • Arthur C. Graesser
}

Published online: 4 January 2013

(C) Psychonomic Society, Inc. 2012

\begin{abstract}
Mind wandering is a phenomenon in which attention drifts away from the primary task to task-unrelated thoughts. Previous studies have used self-report methods to measure the frequency of mind wandering and its effects on task performance. Many of these studies have investigated mind wandering in simple perceptual and memory tasks, such as recognition memory, sustained attention, and choice reaction time tasks. Manipulations of task difficulty have revealed that mind wandering occurs more frequently in easy than in difficult conditions, but that it has a greater negative impact on performance in the difficult conditions. The goal of this study was to examine the relation between mind wandering and task difficulty in a high-level cognitive task, namely reading comprehension of standardized texts. We hypothesized that reading comprehension may yield a different relation between mind wandering and task difficulty than has been observed previously. Participants read easy or difficult versions of eight passages and then answered comprehension questions after reading each of the passages. Mind wandering was reported using the probecaught method from several previous studies. In contrast to the previous results, but consistent with our hypothesis, mind wandering occurred more frequently when participants read difficult rather than easy texts. However, mind wandering had a more negative influence on comprehension for the difficult texts, which is consistent with the previous data. The results are interpreted from the perspectives of the executive-resources and control-failure theories of mind
\end{abstract}

S. Feng $(\bowtie) \cdot$ A. C. Graesser

Psychology Department, University of Memphis,

202 Psychology Building,

Memphis, TN 38152-3230, USA

e-mail: sfeng@memphis.edu

S. D'Mello

University of Notre Dame, Notre Dame, IN, USA wandering, as well as with regard to situation models of text comprehension.

Keywords Mind wandering $\cdot$ Reading comprehension $\cdot$ Text difficulty

Mind wandering is an attentional shift away from the processing of external, task-related information to the processing of internal, task-unrelated information (Smallwood \& Schooler, 2006). Mind wandering involves controlled processing that can occur without intention or even awareness (Schooler, 2002). Recent studies have shown that mind wandering is linked to both the default and executive networks of the brain, thereby suggesting that mind wandering utilizes central executive resources to some extent (Baird, Smallwood, \& Schooler, 2011; Barron, Riby, Greer, \& Smallwood, 2011; Christoff, Gordon, Smallwood, Smith, \& Schooler, 2009). In line with this theory, Smallwood, Schooler, and colleagues have proposed an executive-resource hypothesis, which posits that both task-related and task-unrelated thoughts compete for limited executive resources. When the primary task is difficult and resource demanding, little or no resources are available for mind wandering to occur. On the other hand, mind wandering is more likely to occur when the primary task is easy or its execution has been automated, because it utilizes unused executive resources from the primary task (Smallwood \& Schooler, 2006).

Considerable empirical evidence supports the executiveresource hypothesis, which predicts that mind wandering will be less prominent in difficult than in easy tasks. For example, Smallwood, Obonsawin, and Reid (2003c) had participants perform a fluency task, a verbal-encoding task, or a lottery task. They found that mind wandering increased as a function of block duration in both the verbal-encoding and lottery tasks, but not in the fluency task. Smallwood et 
al. (2003) argued that the fluency task required more attentional resources than did the other tasks, so there was less attentional "space" for mind wandering to occur. Similarly, Smallwood et al. (2004) found that in a sustained-attentionto-response task (Robertson, Manly, Andrade, Baddeley, \& Yiend, 1997), mind wandering was less likely to occur in the first than in the second half of the task, which suggests that mind wandering increased when the task became easier, due to practice effects.

As an alternative to the executive-resource hypothesis, the control-failure hypothesis (McVay \& Kane, 2010) suggests that mind wandering is the result of executive maintenance failures. According to this view, task-related and taskunrelated thoughts frequently compete under executive control, and mind wandering occurs when executive control fails to maintain task-related thoughts by suppressing taskunrelated thoughts. Difficult tasks that require considerable controlled processing to meet the task demands should minimize mind wandering, but only if there are enough cognitive resources and skills to handle the difficult task and if interfering activities are minimal. Individuals with weaker attentional-control abilities are predicted to mind wander more, because of interfering thoughts unrelated to the primary task, than do those with stronger attentionalcontrol abilities (McVay \& Kane, 2012a, 2012b).

In support of this view, McVay and Kane (2012a, 2012b) cited studies that have shown that working memory capacity (WMC) is largely associated with executive-control capabilities (e.g., Kane \& Engle, 2003). Kane et al. (2007) found that people with high WMC were less likely to report mind wandering during attention-demanding activities than were low-WMC individuals. McVay and Kane (2010) hypothesized that if mind wandering competes for working memory resources, as would be predicted by the executive-resource hypothesis, people with high WMC should mind wander more frequently than people with low $\mathrm{WMC}$, because the high-WMC individuals will have more executive resources available for mind wandering. The authors cited empirical data that supported an opposite view, instead characterizing unintended mind wandering as occurring due to a lapse of attentional control rather than to executive-resource availability.

To counter this argument, Levinson, Smallwood, and Davidson (2012) recently conducted a visual-span search study with varying task loads. They found that higher-WMC individuals reported more mind wandering during low task load, but that mind wandering did not depend on WMC during high task load. Levinson et al. interpreted this finding as a challenge to McVay and Kane's (2010) claims, because the McVay and Kane model cannot easily explain why participants with more working memory resources, although better at focusing attention, nonetheless mind wandered more during the low-perceptual-load task.
As is evident from the discussion above, there is considerable debate as to the underlying cognitive processes that give rise to mind wandering. In the present study, we investigated mind wandering in a higher-level cognitive task, namely reading comprehension. The few studies on mind wandering during reading have shown that mind wandering negatively impacts reading comprehension and that comprehension is negatively correlated with probe-caught mind wandering, but not with self-caught mind wandering (Reichle et al., 2009; Smallwood, McSpadden \& Schooler 2008).

One issue that has been addressed unsatisfactorily is the effect of text difficulty on mind wandering. Giambra and Grodsky tracked mind wandering during reading by obtaining human-provided ratings of passage difficulty, and asked readers to rate interest in the passages (Giambra \& Grodsky, 1989; Grodsky \& Giambra, 1990/1991). The authors found that mind wandering was not related to passage difficulty, but was related to readers' interest. However, important limitations of these two studies are that they were not based on objective measures of text difficulty and that the texts varied in content between the difficult and easy passages, which is a potential confound. Giambra and Grodsky also did not report any results linking mind wandering to reading comprehension, or whether mind wandering interacted with text difficulty to predict reading comprehension.

The goal of the present study was to investigate mind wandering during the reading of texts that were equated for content but that varied in difficulty. It is generally acknowledged that reading texts involves the construction of a situation model that represents the most up-to-date interpretation of the text meaning (both explicit information and inferences) and that links the past and present segments of the text (Graesser \& McNamara, 2011; Zwaan, Langston, \& Graesser, 1995). The situation model exerts top-down influence in reading by providing a global context in which a reader interprets the explicit text content and the associated inferences. Difficult texts have more discontinuities in cohesion, which may make it more challenging to maintain sustained control and attention on the comprehension activities. In line with this idea, we expected that mind wandering would occur when a reader had difficulty constructing a situation model of the text (Smallwood, 2011; Smallwood et al., 2008) because of a failure of executive processing, be it due to control failure (McVay and Kane's view) or insufficient executive resources (Smallwood and colleagues' view). Furthermore, mind wandering may disrupt comprehension as a result of the reader's inability to construct a well-formulated situational model of the text (Smallwood, 2011).

Text difficulty in our study was manipulated by creating two versions of a set of texts, instead of having humans rate the difficulty of texts, as was the case in 
the Giambra and Grodsky (Giambra \& Grodsky, 1989; Grodsky \& Giambra, 1990/1991) studies. One goal of this study was to investigate how text difficulty impacts mind wandering. The second goal was to investigate whether text difficulty moderates the impact of mind wandering on reading comprehension.

If mind wandering is affected by difficulty during reading, as it is in the other tasks discussed above, then mind wandering should be systematically influenced by text difficulty. One hypothesis predicts that mind wandering should be more frequent in the easy than in the difficult text condition. This is compatible with Smallwood and Schooler's (2006) executive-resource hypothesis: Difficult tasks utilize more attentional resources, thereby yielding fewer resources available that could allow mind wandering to occur. An alternative hypothesis predicts that mind wandering should be more prominent while reading difficult as compared to easy texts, because readers are likely to experience more difficulty in constructing situation models for the difficult texts.

\section{Method}

Participants

The participants were 80 undergraduate students from a large U.S. university who received course credit for their participation.

\section{Materials}

The texts were eight passages from the Nelson-Denny Reading Comprehension test, version E (Brown, Bennett, \& Hanna, 1981). These eight passages were used in the difficult text condition. Each of the passages was also modified to create an easier version. This was done by simplifying the syntactic structures of sentences and substituting low-frequency words with higher-frequency ones. The average Flesch-Kincaid grade-level scores for the difficult and easy sentences in the texts were 10.9 and 8.9 , respectively, $t(243)=3.706, p<.001$. The average numbers of sentences in the difficult and easy versions of the texts were $13.4(S D=6.89)$ and $17.0(S D=10.64)$, respectively, $t(14)=0.809, p=.432$. The mean numbers of words in the difficult and easy versions of the passages were $261.3(S D=152.2)$ and $255.3(S D=163.7)$, respectively, $t(14)=0.076, p=.941$. These results suggest that our text difficulty manipulation was successful, because it lowered readability while preserving verbosity.

The eight passages and their contents were ordered in the same manner as in the Nelson-Denny Reading Comprehension Test for all participants (Passages 1-8).
There were two sets that counterbalanced whether a text was easy or difficulty. For Set 1 , the difficult passages were the odd-numbered ones (Passages 1, 3, 5, and 7) and the easy passages were the even-numbered ones (Passages 2, 4, 6 , and 8). The opposite assignment of passage difficulties occurred for Set 2. All of the participants read four difficult and four easy passages and were randomly assigned to either Set 1 or 2.

Multiple-choice comprehension questions from the Nelson-Denny test followed every passage. Passage 1 was approximately three times as long as the other passages and had eight comprehension questions. The remaining seven passages had four comprehension questions, thereby yielding a total of 36 comprehension questions. Each text was analyzed to identify information in the text that corresponded to an answer to one of the comprehension questions, and thought probes were inserted at these text positions. The number of probes per text varied from two to seven and was determined by the number and nature of the Nelson-Denny reading comprehension questions. Whole-passage comprehension questions (e.g., "the purpose of this passage is to?") had no thought probes, since answers to these questions could not be linked to a single position in the text. Set 1 had a total of 28 thought probes, and Set 2 had a total of 27 thought probes. The difference in the numbers of thought probes between the two sets was due to how the sentences were split when creating the easy versions of the texts; this discrepancy is addressed in the "Results" section.

\section{Procedure}

The experimental instructions and passages were presented on a computer monitor. Participants first read a definition of mind wandering that was largely taken from previous studies (see Smallwood \& Schooler, 2006, for a review): "Mind wandering is a term used to describe what occurs when your attention wanders from a task. Sometimes when your mind wanders, you begin thinking about personal events or concerns rather than your task. At other times, your mind can wander because you are bored or tired and you don't really know what you're thinking about; all you know is that you are no longer thinking about your task." The participants were then instructed to read each passage one sentence at time, using the spacebar to advance sentences. They could not go back to reread a sentence once they had advanced to the next sentence. Participants were periodically interrupted during reading to respond to a thought probe that read "Were you mind wandering when you read the previous sentence?" They reported whether they had been thinking about the task or having thoughts that had been unrelated to the task by pressing keys labeled "yes" and "no." Reading times were recorded for each sentence. The comprehension questions were presented after each passage. 


\section{Results}

The present analysis focused on sentences in the text that had thought probes that corresponded to the comprehension questions. A total of 2,200 original sentences matched this criterion across all of the participants. Two probes were removed from each participant assigned to Set 1 for Passage 6, and one probe was removed from each participant assigned to Set 2, due to uneven counterbalancing and the ambiguity of the reading comprehension question that corresponded to them. This yielded a set of 2,080 probes after probe removal. The primary dependent measures for each sentence were reading time, mind wandering response ( 0 for no and 1 for yes), and performance on the comprehension question ( 0 for incorrect and 1 for correct) for that sentence.

An examination of the sentence reading time distributions signaled the presence of some outliers that could potentially skew the results. To address this problem, the shortest $1 \%$ (RTs $<395 \mathrm{~ms}$ ) and longest $1 \%$ (RTs > $24,640 \mathrm{~ms}$ ) reading times were replaced with values corresponding to the lowest $(395 \mathrm{~ms})$ and highest $(24,640)$ one percentile, respectively. A total of $41 \mathrm{RTs}-30$ from the difficult and 11 from the easy texts - were replaced by this procedure. Analysis of the RT data both with and without outlier replacement yielded the same results.

A mixed-effects modeling approach was adopted to analyze the data, because of the repeated and nested nature of the design. The lme4 package in R (Bates \& Maechler, 2010) was used to perform the requisite computation. The unit of analysis was an individual sentence, so the data set consisted of 2,080 cases. Linear or logistic models were constructed on the basis of whether the dependent variable was continuous (reading times) or binary (mind wandering and reading comprehension), respectively. The random effects were participants (80 levels) and sentences, which had a unique number to identify each sentence with a thought probe (26 levels). Condition was a two-level (difficult and easy) categorical fixed effect, with the easy condition set as the reference group. An alpha value of .05 was adopted for all significance testing, and two-tailed tests were used.

\section{Reading times}

A mixed-effects linear regression model using text difficulty to predict sentence reading times was significant, $F(1,2078)$ $=150, p<.001$. We found a 1,544-ms (i.e., $B=1,544, S E=$ $126, p<.001)$ increase in reading times when participants read the difficult $(M=7,164, S D=2,719)$ versus the easy $(M=5,625, S D=1,914)$ versions of the sentences. This provides some additional evidence in support of our text difficulty manipulation.
Mind-wandering frequency

A mixed-effects logistic regression model for the presence (coded as 1) or absence (coded as 0) of mind wandering, with text difficulty as a fixed effect, yielded a significantly better fit than did a model with only the random effect but without the fixed effect, $\chi^{2}(1)=4.49, p<.05$. The coefficient was $0.212(S E=.098, p=.031)$, which indicates that participants were $1.24\left(\mathrm{e}^{0.212}\right)$ times more likely to mind wander when reading a difficult than when reading an easy sentence. The mean proportions of mind wandering were .423 for the difficult texts and .362 for the easy texts.

There was the concern that increased mind wandering in the difficult condition might be confounded by the longer reading times for the probe sentences in the difficult texts, because longer tasks provide more opportunities for mind wandering than do easier tasks. Fortunately, the overall reading times (in minutes) for the four difficult texts $(M=$ $5.50, S D=2.26$ ) were not statistically different from the overall reading times for the four easy texts $(M=5.25, S D=$ $2.14), t(79)=0.727, p=.470)$. In addition, Spearman's correlations between overall reading times and the proportions of mind wandering were very small and not significant $(r=-.053, p=.640$, for the difficult texts; $r=.108, p=.341$, for the easy texts), suggesting that, overall, reading time was not related to mind wandering.

Additionally, our findings cannot be attributed to simple fatigue effects, because (1) easy and difficult texts were interleaved; (2) the average task time was 18 min $13 \mathrm{~s}(S D$ $=4 \min 23 \mathrm{~s}$ ), which is quite short; and (3) text order (i.e., first, second, third, etc.) did not correlate with the proportions of mind wandering (Spearman's $r=.038, p=.337$ ).

\section{Reading comprehension}

On average, participants answered $61.9 \%$ of the questions correctly for the easy texts, and $56.2 \%$ for the difficult texts. A mixed-effects logistic regression model for correct (coded as 1) versus incorrect (coded as 0 ) responses on the comprehension test was not significant, $\chi^{2}(1)=3.16, p=.075$. Participants were 1.20 times $(B=-.185, S E=.103, p=$ $.075)$ less likely to answer correctly on a comprehension test item when they had read the difficult versus the easy version of the text. This effect was not statistically significant with respect to an alpha of .05 , and therefore should be taken as a tentative finding.

Mind wandering, reading times, and reading comprehension

We investigated whether mind wandering predicted reading times by regressing sentence reading times on the presence or absence of mind wandering (for that sentence). The resultant mixed-effects linear regression model was 
significant, $F(1,2078)=41.4, p<.001$ : Reading times increased by $958 \mathrm{~ms}$ when participants were mind wandering, as compared to when they were on task $(B=958, S E=$ $149, p<.001)$. Mind wandering also predicted reading times after controlling for condition (i.e., including condition as a fixed effect), $F(1,2077)=36.9, p<.001, B=875, S E=$ 144 . We also investigated whether mind wandering moderated the effect of text difficulty on reading times by including the Mind Wandering $\times$ Text Difficulty interaction term as a fixed effect. However, this did not yield a significant model, $B=-34.89, S E=40.92, F$ $(1,2076)=0.727, p=.394$.

A logistic regression model that regressed response correctness for comprehension questions on mind wandering was significant, $\chi^{2}(1)=11.3, p<.001$. Participants were 1.5 times less likely $(B=-.387, S E=.113, p<.001)$ to respond correctly to a comprehension question on a sentence if they had mind wandered while reading that sentence.

Finally, we investigated whether mind wandering moderated the effect of text difficulty on comprehension scores by including the Mind Wandering $\times$ Text Difficulty interaction term as a fixed effect. The interaction was significant $[B=$ $.110, S E=.039, F(1,2076)=7.716, p<.01]$ and is depicted in Fig. 1 . The interaction was probed by regression comprehension scores on mind wandering separately for the easy versus the difficult texts. The results indicated that mind wandering did not negatively influence reading comprehension for the easy texts $(B=-.143, S E=.160, p=.373)$ but had a substantial negative influence for the difficult texts $(B=$ $-.625, S E=.158, p<.001)$. Indeed, participants were 1.87 times less likely to answer correctly on an item on the

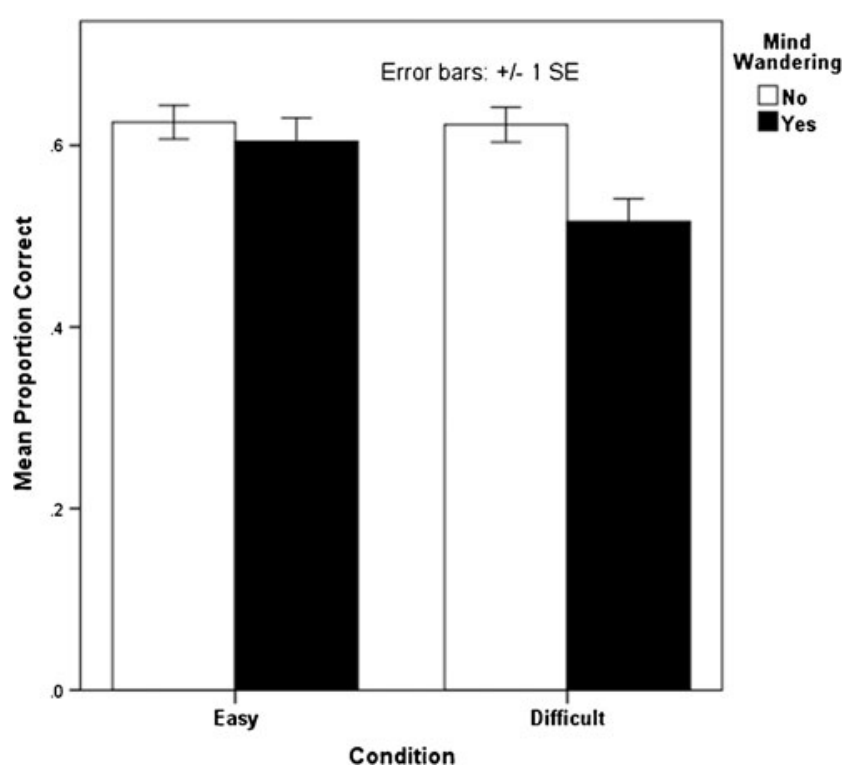

Fig. 1 Mind wandering and comprehension as a function of text difficulty. comprehension test if they had mind wandered while reading a difficult text on that item.

\section{Discussion}

The goal of this experiment was to examine text comprehension as a function of mind wandering and text difficulty. In this section, we align the findings with our hypotheses, discuss limitations, and consider extensions of future research.

\section{Major findings}

The results indicated that mind wandering interacted with text difficulty in predicting reading comprehension. Specifically, mind wandering had a substantial negative effect on comprehension when the text was difficult to comprehend. This finding is consistent with Smallwood and Schooler's (2006) executive-resource hypothesis, which posits that mind wandering impairs performance more when the primary task is difficult. However, the present study indicated that mind wandering was more frequent in the difficult than in the easy condition, which is inconsistent with previous studies that have found that task difficulty is associated with a reduction in the frequency of mind wandering. It is also contrary to Grodsky and Giambra's (Giambra \& Grodsky, 1989; Grodsky \& Giambra, 1990/ 1991) finding that passage difficulty was not related to mind wandering.

The finding that there was more mind wandering in the difficult condition can be best explained by the situationmodel view, which posits that mind wandering increases when readers have difficulty constructing a situation model from the text (Smallwood, 2011; Smallwood et al., 2008). Specifically, when a text affords the construction of a situation model, attentional resources and text comprehension processes work in tandem. Attentional resources that attend to the primary task can suppress off-task thoughts that are competing for attention. The longer reading times and lower, albeit not significantly $(p=.075)$, comprehension scores associated with the difficult text provide some tentative evidence that participants were less successful in constructing situation models when reading these texts, which might lead to more mind wandering.

In this study, we also showed that reading times were longer when participants' minds wandered than when they were on task. Previous research involving low-level perceptual tasks has found that reaction times are often faster when participants mind wander than when they are on-task (e.g., Smallwood et al., 2004). However, reaction times are often slow when participants mind wander in actively engaging tasks, such as word encoding (Smallwood, Baraciaia, Lowe, 
\& Obonsawin, 2003; Smallwood, Obonsawin, \& Heim, 2003) and, in our study, a high-level processing task of reading.

Limitations and future directions

Some potential limitations of our study warrant further investigation. First, we did not investigate the role of interest. The lack of a measure of interest might be a limitation because previous studies have shown that interest is negatively related to mind wandering (Giambra \& Grodsky, 1989; Grodsky \& Giambra, 1990/1991; Smallwood, Nind, \& O'Connor, 2009). It might be that interest in a text has a compensatory effect on the relation between text difficulty and mind wandering. Mind wandering may occur more often while reading difficult texts, but only among noninterested participants. The question of whether interest moderates the effect of text difficulty on mind wandering awaits future research.

Another consideration is that our task was a sentence-bysentence self-paced task, which may deviate from normal reading tasks in which the reader has the entire referential context in view during reading. For future studies, it would be helpful to assess whether text difficulty predicts mind wandering after controlling for differences in styles of text presentation. Reading tasks that are timed versus self-paced may also impact mind wandering; when participants are pressed for time, they may make more effort to comprehend the difficult texts than if they are reading at their own pace. Experimenter-paced presentations of text may reduce mind wandering because readers would need to allocate more attentional resources in order to successfully comprehend the texts, due to time constraints. In future experiments, it would be interesting to investigate whether the data would show the same pattern with timed tasks.

Author note This research was supported by the National Science Foundation (NSF; Grant Nos. ITR 0325428, HCC 0834847, and DRL 1235958). Any opinions, findings, conclusions, or recommendations expressed in this article are those of the authors and do not necessarily reflect the views of the NSF. Our special gratitude goes to Debra Long for her guidance in the development of the present experimental design.

\section{References}

Baird, B., Smallwood, J., \& Schooler, J. W. (2011). Back to the future: Autobiographical planning and the functionality of mindwandering. Conscious and Cognition, 20, 1604-1611. doi:10.1016/j.concog.2011.08.007

Barron, E., Riby, L. M., Greer, J., \& Smallwood, J. (2011). Absorbed in thought: The effect of mind wandering on the processing of relevant and irrelevant events. Psychological Science, 22, 596601. doi:10.1177/0956797611404083
Bates, D. M., \& Maechler, M. (2010). lme4: Linear mixed-effects models using S4 classes [Software]. Retrieved from http:// cran. $r$-project.org/web/packages/lme4/index.html

Brown, J. I., Bennett, J. M., \& Hanna, G. (1981). The Nelson-Denny reading test. Chicago, IL: Riverside.

Christoff, K., Gordon, A. M., Smallwood, J., Smith, R., \& Schooler, J. W. (2009). Experience sampling during fMRI reveals default network and executive system contributions to mind wandering. Proceedings of the National Academy of Sciences, 106, 87198724. doi:10.1073/pnas.0900234106

Giambra, L. M., \& Grodsky, A. (1989). Task-unrelated images and thoughts while reading. In J. E. Shorr, P. Robin, J. A. Connella, \& M. Wolpin (Eds.), Imagery: Current perspectives (pp. 27-32). New York, NY: Plenum Press.

Graesser, A. C., \& McNamara, D. S. (2011). Computational analysis of multilevel discourse comprehension. Topics in Cognitive Science, 3, 371-398. doi:10.1111/j.1756-8765.2010.01081.x

Grodsky, A., \& Giambra, L. (1990/1991). The consistency across vigilance and reading tasks of individual differences in the occurrence of task unrelated and task related images and words. Imagination, Cognition and Personality, 10, 39-52.

Kane, M. J., Brown, L. H., McVay, J. C., Silvia, P. J., Myin-Germeys, I., \& Kwapil, T. R. (2007). For whom the mind wanders, and when: An experience sampling study of working memory and executive control in everyday life. Psychological Science, 18, 614-621. doi:10.1111/j.1467-9280.2007.01948.x

Kane, M. J., \& Engle, R. W. (2003). Working-memory capacity and the control of attention: The contributions of goal neglect, response competition, and task set to Stroop interference. Journal of Experimental Psychology: General, 132, 47-70. doi:10.1037/ 0096-3445.132.1.47

Levinson, D. B., Smallwood, J., \& Davidson, R. J. (2012). The persistence of thought: Evidence for a role of working memory in the maintenance of task-unrelated thinking. Psychological Science, 23, 375-380. doi:10.1177/0956797611431465

McVay, J. C., \& Kane, M. J. (2010). Does mind wandering reflect executive function or executive failure? Comment on Smallwood and Schooler (2006) and Watkins (2008). Psychological Bulletin, 136, 188-197. doi:10.1037/a0018298

McVay, J. C., \& Kane, M. J. (2012a). Drifting from slow to “ $d$ 'oh!": Working memory capacity and mind wandering predict extreme reaction times and executive control errors. Journal of Experimental Psychology: Learning, Memory, and Cognition, 38, 525-549. doi:10.1037/a0025896

McVay, J. C., \& Kane, M. J. (2012b). Why does working memory capacity predict variation in reading comprehension? On the influence of mind wandering and executive attention. Journal of Experimental Psychology: General, 141, 302-320. doi:10.1037/a0025250

Reichle, E. D., Morales, F. J., Laurent, P. A., Halpern, D. V., Smallwood, J., \& Schooler, J. W. (2009). Mindless reading: The monitoring and impact of mind-wandering during reading. Unpublished manuscript

Robertson, I. H., Manly, T., Andrade, J., Baddeley, B. T., \& Yiend, J. (1997). "Oops!": Performance correlates of everyday attentional failures in traumatic brain injured and normal subjects. Neuropsychologia, 35, 747-758. doi:10.1016/S0028-3932 (97)00015-8

Schooler, J. W. (2002). Re-representing consciousness: Dissociations between experience and meta-consciousness. Trends in Cognitive Sciences, 6, 339-344. doi:10.1016/S1364-6613(02)01949-6

Smallwood, J. (2011). Mind-wandering while reading: Attentional decoupling, mindless reading and the cascade model of inattention. Language and Linguistics Compass, 5, 63-77. doi:10.1111/ j.1749-818x.2010.00263.x

Smallwood, J., Baraciaia, S. F., Lowe, M., \& Obonsawin, M. C. (2003a). Task unrelated thought whilst encoding information. 
Consciousness and Cognition, 12, 452-484. doi:10.1016/S10538100(03)00018-7

Smallwood, J., Davies, J. B., Heim, D., Finnigan, F., Sudberry, M., O'Connor, R., \& Obonsawin, M. (2004). Subjective experience and the attentional lapse: Task engagement and disengagement during sustained attention. Consciousness and Cognition, 4, 657690. doi:10.1016/j.concog.2004.06.003

Smallwood, J., McSpadden, M., \& Schooler, J. W. (2008). When attention matters: The curious incident of the wandering mind. Memory \& Cognition, 36, 1144-1150. doi:10.3758/MC.36.6.1144

Smallwood, J., Nind, L., \& O'Connor, R. C. (2009). When is your head at? An exploration of the factors associated with the temporal focus of the wandering mind. Consciousness and Cognition, 18, 118-125. doi:10.1016/j.concog.2008.11.004
Smallwood, J., Obonsawin, M. C., \& Heim, S. D. (2003b). Task unrelated thought: The role of distributed processing. Consciousness and Cognition, 11, 169-189. doi:10.1016/S10538100(02)00003-X

Smallwood, J., Obonsawin, M., \& Reid, H. (2003c). The effects of block duration and task demands on the experience of task unrelated thought. Imagination, Cognition and Personality, 22, 13-31. doi:10.2190/TBML-N8JN-W5YB-4L9R

Smallwood, J., \& Schooler, J. W. (2006). The restless mind. Psychological Bulletin, 132, 946-958. doi:10.1037/0033-2909.132.6.946

Zwaan, R. A., Langston, M. C., \& Graesser, A. C. (1995). The construction of situation models in narrative comprehension: An event-indexing model. Psychological Science, 6, 292-297. doi:10.1111/j.1467-9280.1995.tb00513.x 